\title{
Does chocolate reduce blood pressure? A meta-analysis
}

\author{
Karin Ried ${ }^{1 *}$, Thomas Sullivan ${ }^{2}$, Peter Fakler ${ }^{1}$, Oliver R Frank¹, Nigel P Stocks ${ }^{1}$
}

\begin{abstract}
Background: Dark chocolate and flavanol-rich cocoa products have attracted interest as an alternative treatment option for hypertension, a known risk factor for cardiovascular disease. Previous meta-analyses concluded that cocoa-rich foods may reduce blood pressure. Recently, several additional trials have been conducted with conflicting results. Our study summarises current evidence on the effect of flavanol-rich cocoa products on blood pressure in hypertensive and normotensive individuals.
\end{abstract}

Methods: We searched Medline, Cochrane and international trial registries between 1955 and 2009 for randomised controlled trials investigating the effect of cocoa as food or drink compared with placebo on systolic and diastolic blood pressure (SBP/DBP) for a minimum duration of 2 weeks. We conducted random effects meta-analysis of all studies fitting the inclusion criteria, as well as subgroup analysis by baseline blood pressure (hypertensive/ normotensive). Meta-regression analysis explored the association between type of treatment, dosage, duration or baseline blood pressure and blood pressure outcome. Statistical significance was set at $P<0.05$.

Results: Fifteen trial arms of 13 assessed studies met the inclusion criteria. Pooled meta-analysis of all trials revealed a significant blood pressure-reducing effect of cocoa-chocolate compared with control (mean BP change \pm SE: SBP: $-3.2 \pm 1.9 \mathrm{mmHg}, P=0.001 ; \mathrm{DBP}:-2.0 \pm 1.3 \mathrm{mmHg}, P=0.003)$. However, subgroup meta-analysis was significant only for the hypertensive or prehypertensive subgroups (SBP: $-5.0 \pm 3.0 \mathrm{mmHg} ; P=0.0009$; DBP: $-2.7 \pm$ $2.2 \mathrm{~mm} \mathrm{Hg}, P=0.01$ ), while BP was not significantly reduced in the normotensive subgroups (SBP: $-1.6 \pm 2.3$ $\mathrm{mmHg}, P=0.17$; DBP: $-1.3 \pm 1.6 \mathrm{mmHg}, P=0.12$ ). Nine trials used chocolate containing $50 \%$ to $70 \%$ cocoa compared with white chocolate or other cocoa-free controls, while six trials compared high- with low-flavanol cocoa products. Daily flavanol dosages ranged from $30 \mathrm{mg}$ to $1000 \mathrm{mg}$ in the active treatment groups, and interventions ran for 2 to 18 weeks. Meta-regression analysis found study design and type of control to be borderline significant but possibly indirect predictors for blood pressure outcome.

Conclusion: Our meta-analysis suggests that dark chocolate is superior to placebo in reducing systolic hypertension or diastolic prehypertension. Flavanol-rich chocolate did not significantly reduce mean blood pressure below $140 \mathrm{mmHg}$ systolic or $80 \mathrm{mmHg}$ diastolic.

\section{Background}

Flavanol-rich chocolate and cocoa products have attracted interest as nonpharmacological treatment options for high blood pressure, a known risk factor for cardiovascular disease $[1,2]$. Even small reductions in blood pressure substantially reduce cardiovascular risk $[3,4]$. Current guidelines strongly recommend integration of lifestyle modification and complementary treatment with the use of conventional blood pressure medications [5].

\footnotetext{
* Correspondence: karin.ried@adelaide.edu.au

'Discipline of General Practice, The University of Adelaide, Adelaide, SA 5005, Australia
}

(c) 2010 Ried et al; licensee BioMed Central Ltd. This is an Open Access article distributed under the terms of the Creative Commons Attribution License (http://creativecommons.org/licenses/by/2.0), which permits unrestricted use, distribution, and reproduction in any medium, provided the original work is properly cited. have been shown to increase the formation of endothelial nitric oxide, which promotes vasodilation and consequently may lower blood pressure [6-8]. Meta-analyses by Taubert et al. (2007) [9], including five small trials [10-13], and Desch et al. (2010) [14], including 10 trials [10-13,15-20], concluded that cocoa-rich foods may reduce blood pressure. However, additional trials have been published since then, with conflicting results [21-24]. Our study updates current research on the effect of cocoa-rich products taken daily for two or more weeks compared with placebo on blood pressure 
in adults. In addition, we explored the influence of baseline blood pressure, dosage, duration, type of control, study design, age, body mass index and trial quality on blood pressure outcome.

\section{Methods}

\section{Search strategy}

We searched the Medline and Cochrane databases for randomised controlled trials of chocolate or cocoa on blood pressure published between 1955 and 2009 using the following search terms: chocolate OR cocoa AND blood pressure. We also searched reference lists of published studies and checked international trial registries http://www.clinicaltrials.gov; http://www.trialregister.nl; http://www.anzctr.org.au; http://www.controlled-trials. com for unpublished but completed studies investigating chocolate/cocoa for blood pressure.

\section{Selection of trials}

Trials were included in the meta-analysis if the control group received a placebo or a low dose of flavanolcontaining cocoa product (drink, bar or tablet), the trial duration was $\geq 14$ days, and the clinical mean or median systolic or diastolic blood pressure (SBP/DBP) and standard deviation (SD) were available. We contacted authors of studies which did not report numerical mean SBP/DBP or SD and received datasets from two studies $[18,22]$, which we included in the meta-analysis. Three eligible completed but unpublished studies were excluded because data were not available at the time of this study [25-27].

\section{Data extraction and quality assessment}

Data were abstracted and quality was assessed independently by two investigators (KR, PF) using guidelines published by the Cochrane Collaboration [28] (Tables $1,2,3)$. Any disagreement was resolved by discussion between the authors (KR, PF) in consultation with the statistician (TS). Characteristics of trials included in the meta-regression analysis are shown in Table 1 . We assessed quality on the basis of randomisation, blinding, whether blood pressure was a primary outcome measure, loss to follow-up, funding source and whether compliance and dietary chocolate intake had been assessed, as these could have influenced findings (Table $3)$. No trial was excluded in the meta-analysis on grounds of quality; however, higher-quality trials (score $\geq 3.5$ of 5 points) were compared with lower-quality trials by meta-regression analysis.

\section{Analysis}

Meta-analysis was conducted using the Cochrane Program Review Manager version 5 [29]. Owing to high heterogeneity between trials, we used a random effects model and considered subgroup meta-analysis by baseline mean blood pressure, similar to our recent metaanalysis of the effect of garlic on blood pressure [30]. For systolic blood pressure, trials were divided into a hypertensive subgroup ( $\mathrm{SBP} \geq 140 \mathrm{mmHg}$ ) and a normotensive subgroup (SBP $<140 \mathrm{mmHg}$ ) at the start of treatment. For diastolic blood pressure, a division into a higher BP subgroup (DBP $\geq 80 \mathrm{mmHg}$ ) and lower BP subgroup (DBP $<80 \mathrm{mmHg}$ ) at the start of treatment allowed an even distribution of trials between subgroups and reduction in heterogeneity.

Meta-regression analyses were conducted using Stata version 10 [31] to explore reasons for high heterogeneity in the pooled meta-analysis of all studies. The following variables were tested, as their associations with blood pressure outcomes are physiologically plausible: Dosage of polyphenols in the active treatment group (continuous variable), type of control (categorical variable: low-flavanol control as drink, tablet or bar/ flavanol-free control as white chocolate, milk, or placebo capsules), duration (continuous and categorical $>2$ weeks yes/no), study design (parallel versus crossover), starting SBP (continuous and categorical > $140 \mathrm{mmHg}$ yes/no), starting DBP (continuous and categorical $>80$ $\mathrm{mmHg}$ yes/no), quality score ( $\geq 3.5 \mathrm{yes} / \mathrm{no})$, average body mass index (BMI) (continuous and categorical > 25 or $>30$ yes/no) and average age (continuous).

If meta-regression results indicated a variable to contribute significantly to heterogeneity between studies, subgroup analysis by this variable was conducted, testing whether there was an effect of treatment on blood pressure outcomes within each subgroup. If heterogeneity was reduced, the subgroup analysis provided a more reliable estimate of pooled effect size between the treatment groups. Additionally, sensitivity analysis excluding selected trials explored the robustness of results. Publication bias or small study effect was assessed by Begg's funnel plots and Egger's regression tests [32,33].

\section{Results}

\section{Summary of included studies}

A total of 18 publications including 21 trial arms were assessed in detail for inclusion [10-13,15-24,34-38] (Figure 1). Fifteen trial arms reported in 13 publications met the inclusion criteria [10-13,15-18,20-24] (Figure 1, Table 1). Six trial arms were excluded because 1) the same population and protocol were used in [19] compared with [13]; 2) the comparison group received other vasoactive substances rather than placebos as a) chocolate \pm plant sterols $[34,35]$, b) tomato extract in phase 2 of trial [23], or c) half dose of chocolate [38]; 3) mean SBP/DBP and SD were not reported and could not be obtained from the authors [36]; and 4) the trial was of 1-day duration [37] (Table 2). 
Table 1 Characteristics of trials included in the meta-analysis

\begin{tabular}{|c|c|c|c|c|c|c|c|}
\hline Trial; Location & $\begin{array}{l}\text { Study design; } \\
\text { treatment/control } \\
\text { groups }\end{array}$ & $\begin{array}{l}\text { Dosage; } \\
\text { duration }\end{array}$ & $\begin{array}{l}\text { Active } \\
\text { ingredients per } \\
\text { daily dosage }\end{array}$ & $\begin{array}{l}\text { Mean } \\
\text { age; } \\
\text { mean } \\
\text { BMI }\end{array}$ & $\begin{array}{l}\mathrm{N} \\
\text { treatment/ } \\
\text { control }\end{array}$ & $\begin{array}{l}\text { Mean SBP (SD) in } \\
\text { mmHg at start/end of } \\
\text { treatment versus } \\
\text { control }\end{array}$ & $\begin{array}{l}\text { Mean DBP (SD) in } \\
\text { mmHg at start/end of } \\
\text { treatment versus } \\
\text { control }\end{array}$ \\
\hline \multirow[t]{2}{*}{$\begin{array}{l}\text { Taubert et al. } \\
\text { [10]; Germany }\end{array}$} & Crossover $^{a, b}$ & 100 g/d; & $\begin{array}{l}500 \mathrm{mg} \\
\text { polyphenols }\end{array}$ & $59.5 \mathrm{yr} ;$ & $13 / 13$ & $\begin{array}{l}\text { Cocoa: } 153.4(4.4) / 148.6 \\
(2.4) ;\end{array}$ & $\begin{array}{l}\text { Cocoa: } 84.5(4.6) / 82.9 \\
(4.6) ;\end{array}$ \\
\hline & $\begin{array}{l}\text { Dark/white } \\
\text { chocolate }\end{array}$ & $14 d$ & & 24.1 & & $\begin{array}{l}\text { Control: } 153.6(4.4) / \\
154.0(3.6)\end{array}$ & $\begin{array}{l}\text { Control: } 84.2(4.2) / 84.5 \\
(4.3)\end{array}$ \\
\hline \multirow[t]{2}{*}{$\begin{array}{l}\text { Murphy et al. } \\
\text { [15]; Australia }\end{array}$} & Parallel; & 6 tablets/d & $\begin{array}{l}234 \mathrm{mg} \\
\text { flavanols; }\end{array}$ & $43.5 \mathrm{yr} ;$ & $13 / 15$ & $\begin{array}{l}\text { Cocoa: } 118 \text { (13)/120 } \\
(12) ;\end{array}$ & Cocoa: 78 (12)/77 (10); \\
\hline & $\begin{array}{l}\text { High/low flavanol } \\
\text { cocoa tablets }\end{array}$ & $28 d$ & Control: 6.4 mg & 26.0 & & Control: 116 (9)/119 (8) & Control: 76 (8)/76 (7) \\
\hline \multirow[t]{2}{*}{$\begin{array}{l}\text { Engler [11]; } \\
\text { USA }\end{array}$} & Parallel; & 46 g/d; & $\begin{array}{l}213 \mathrm{mg} \\
\text { procyanidins }\end{array}$ & $32.1 \mathrm{yr} ;$ & $11 / 10$ & $\begin{array}{l}\text { Cocoa: } 121 \text { (17.9)/120 } \\
(13.3)\end{array}$ & $\begin{array}{l}\text { Cocoa: } 68.1(8.3) / 69 \\
(6.6)\end{array}$ \\
\hline & $\begin{array}{l}\text { High/low flavanoid } \\
\text { chocolate }\end{array}$ & $14 d$ & & 22.6 & & $\begin{array}{l}\text { Control: } 112.8(8.9) / 110 \\
(6.3)\end{array}$ & $\begin{array}{l}\text { Control: } 66.1(5.4) / 66 \\
(6.3)\end{array}$ \\
\hline \multirow[t]{2}{*}{$\begin{array}{l}\text { Fraga et al. } \\
\text { [12]; USA }\end{array}$} & Crossover $^{\mathrm{a}}$ & 105 g/d; & $\begin{array}{l}168 \mathrm{mg} \\
\text { flavanols }\end{array}$ & $18.0 \mathrm{yr} ;$ & $27 / 28$ & $\begin{array}{l}\text { Cocoa: } 123(11.2) / 117 \\
(7.5)\end{array}$ & Cocoa: 72 (7.5)/67 (7.5) \\
\hline & $\begin{array}{l}\text { Dark/white } \\
\text { chocolate }\end{array}$ & $14 d$ & & 24.1 & & $\begin{array}{l}\text { Control: } 123(11.2) / 121 \\
(7.5)\end{array}$ & Control: 71 (7.5)/70 (7.5) \\
\hline \multirow{2}{*}{$\begin{array}{l}\text { Grassi } \\
\text { (normotensive } \\
\text { arm) [13]; Italy }\end{array}$} & Crossover $^{\mathrm{a}, \mathrm{b}}$ & 100 g/d; & $\begin{array}{l}500 \mathrm{mg} \\
\text { polyphenols }\end{array}$ & $33.9 \mathrm{yr} ;$ & $15 / 15$ & $\begin{array}{l}\text { Cocoa: } 112.9(8.5) / 105.9 \\
(6.6)\end{array}$ & $\begin{array}{l}\text { Cocoa: } 74.0(5.7) / 69.8 \\
(4.5)\end{array}$ \\
\hline & $\begin{array}{l}\text { Dark/white } \\
\text { chocolate }\end{array}$ & $15 \mathrm{~d}$ & & 22.6 & & $\begin{array}{l}\text { Control: } 113.2(7.9) / \\
112.7(7.6)\end{array}$ & $\begin{array}{l}\text { Control: } 73.8(5.5) / 73.5 \\
(5.3)\end{array}$ \\
\hline \multirow{2}{*}{$\begin{array}{l}\text { Grassi et al. } \\
\text { (hypertensive } \\
\text { arm [13]; Italy }\end{array}$} & Crossover $^{\mathrm{a}, \mathrm{b}}$ & 100 g/d; & $\begin{array}{l}500 \mathrm{mg} \\
\text { polyphenols }\end{array}$ & $43.7 \mathrm{yr} ;$ & $20 / 20$ & $\begin{array}{l}\text { Cocoa: } 141.3(4.8) / 129.3 \\
(5.7) ;\end{array}$ & $\begin{array}{l}\text { Cocoa: } 92.4(3.8) / 84.6 \\
(5.6) ;\end{array}$ \\
\hline & $\begin{array}{l}\text { Dark/white } \\
\text { chocolate }\end{array}$ & $15 \mathrm{~d}$ & & 25.4 & & $\begin{array}{l}\text { Control: } 141.1 \text { (5.4)/ } \\
140.4(4.6)\end{array}$ & $\begin{array}{l}\text { Control: } 91.8 \text { (4.7)/91.2 } \\
(4.7)\end{array}$ \\
\hline \multirow[t]{2}{*}{$\begin{array}{l}\text { Taubert et al. } \\
\text { [16]; Germany }\end{array}$} & Parallel $^{\mathrm{a}}$; & $6.3 \mathrm{~g} / \mathrm{d}$ & $\begin{array}{l}30 \mathrm{mg} \\
\text { polyphenols }\end{array}$ & $63.6 \mathrm{yr} ;$ & $22 / 22$ & $\begin{array}{l}\text { Cocoa: } 147.7(7.1) / 144.8 \\
\text { (ng); }\end{array}$ & $\begin{array}{l}\text { Cocoa: } 86.4(4.1) / 84.5 \\
\text { (ng); }\end{array}$ \\
\hline & $\begin{array}{l}\text { Dark/white } \\
\text { chocolate }\end{array}$ & $126 \mathrm{~d}(18 w \mathrm{w})$ & & 24.0 & & $\begin{array}{l}\text { Control: } 147.5 \text { (8.0)/ } \\
147.6 \text { (ng) }\end{array}$ & $\begin{array}{l}\text { Control: } 86.7 \text { (3.8)/86.7 } \\
\text { (n.g.) }\end{array}$ \\
\hline \multirow[t]{2}{*}{$\begin{array}{l}\text { Crews et al. } \\
\text { [17]; USA }\end{array}$} & Parallel $^{\mathrm{a}}$; & $\begin{array}{l}37 \mathrm{~g} / \mathrm{d} \text { choc }+ \\
11 \mathrm{~g} / \mathrm{d} \text { drink; }\end{array}$ & $\begin{array}{l}754 \mathrm{mg} \\
\text { proanthocyanins; }\end{array}$ & $68.8 \mathrm{yr} ;$ & $45 / 45$ & $\begin{array}{l}\text { Cocoa: } 126.8(14.3) / \\
123.3(12.3) ;\end{array}$ & $\begin{array}{l}\text { Cocoa: } 74.2(8.2) / 73.7 \\
\text { (7.5); }\end{array}$ \\
\hline & $\begin{array}{l}\text { Dark chocolate }+ \\
\text { cocoa drink/low } \\
\text { flavanol chocolate }+ \\
\text { drink }\end{array}$ & $42 d(6 w k)$ & Control: $41.1 \mathrm{mg}$ & 25.3 & & $\begin{array}{l}\text { Control: } 128.6(14.3) / \\
125.5 \text { (12.7) }\end{array}$ & $\begin{array}{l}\text { Control: } 75.0(8.0) / 74.4 \\
(7.7)\end{array}$ \\
\hline \multirow[t]{2}{*}{$\begin{array}{l}\text { Grassi et al. } \\
\text { [18]; Italy }\end{array}$} & Crossover $^{a, b}$ & 100 g/d; & $\begin{array}{l}1008 \mathrm{mg} \\
\text { phenols }\end{array}$ & $44.8 \mathrm{yr} ;$ & $19 / 19$ & $\begin{array}{l}\text { Cocoa: } 141.1(3.4) / 137.3 \\
(4.0) ;\end{array}$ & $\begin{array}{l}\text { Cocoa: } 91.2(4.2) / 87.3 \\
(4.6) ;\end{array}$ \\
\hline & $\begin{array}{l}\text { Dark/white } \\
\text { chocolate }\end{array}$ & $15 \mathrm{~d}$ & & 26.5 & & $\begin{array}{l}\text { Control: } 140.9(3.4) / \\
140.8 \text { (3.5) }\end{array}$ & $\begin{array}{l}\text { Control: } 91.1 \text { (3.7)/90.9 } \\
(3.5)\end{array}$ \\
\hline \multirow[t]{2}{*}{$\begin{array}{l}\text { Muniyappa et } \\
\text { al. [20]; USA }\end{array}$} & Crossover $^{a, b}$ & 31 g/d cocoa; & $\begin{array}{l}900 \text { mg } \\
\text { polyphenols; }\end{array}$ & $51.0 \mathrm{yr} ;$ & $20 / 20$ & Cocoa: 141 (13)/139 (9); & $\begin{array}{l}\text { Cocoa: } 91 \text { (13) } \\
88(9) ;\end{array}$ \\
\hline & $\begin{array}{l}\text { High flavanol } \\
\text { cocoa/low flavanol } \\
\text { drink }\end{array}$ & $14 \mathrm{~d}$ & Control: 28 mg & 33.2 & & $\begin{array}{l}\text { Control: } 141 \text { (13)/140 } \\
\text { (9) }\end{array}$ & Control: 91 (13)/87 (9) \\
\hline \multirow{2}{*}{$\begin{array}{l}\text { Davison et al. } \\
\text { (nonexercise } \\
\text { arm) [21]; } \\
\text { Australia }\end{array}$} & Parallel; & $\begin{array}{l}300 \mathrm{ml} \text { drink } \\
\mathrm{mix} / \mathrm{d}\end{array}$ & $\begin{array}{l}902 \mathrm{mg} \\
\text { flavanols; }\end{array}$ & $44.9 \mathrm{yr} ;$ & $12 / 11$ & $\begin{array}{l}\text { Cocoa: } 124(10.4) / 122.1 \\
\text { (ng); }\end{array}$ & $\begin{array}{l}\text { Cocoa: } 76(6.2) / 74.2 \\
(\mathrm{ng}) ;\end{array}$ \\
\hline & $\begin{array}{l}\text { High flavanol } \\
\text { cocoa/low flavanol } \\
\text { drink }\end{array}$ & $84 \mathrm{~d}(12 \mathrm{wk})$ & Control: 36 mg & 33.6 & & $\begin{array}{l}\text { Control: } 124(5.97) / \\
128.2 \text { (ng) }\end{array}$ & $\begin{array}{l}\text { Control: } 77 \text { (5.0)/79.8 } \\
\text { (ng) }\end{array}$ \\
\hline $\begin{array}{l}\text { Davison } \\
\text { (exercise arm) } \\
\text { [21]; Australia }\end{array}$ & Parallel; & $\begin{array}{l}300 \mathrm{ml} \text { drink } \\
\text { mix/d; }\end{array}$ & $\begin{array}{l}902 \mathrm{mg} \\
\text { flavanols; }\end{array}$ & $45.4 \mathrm{yr} ;$ & $13 / 13$ & $\begin{array}{l}\text { Cocoa: } 126(10.4) / 127.1 \\
\text { (ng); }\end{array}$ & $\begin{array}{l}\text { Cocoa: } 78(8.7) / 77.5 \\
\text { (ng); }\end{array}$ \\
\hline
\end{tabular}


Table 1: Characteristics of trials included in the meta-analysis (Continued)

\begin{tabular}{|c|c|c|c|c|c|c|c|}
\hline & $\begin{array}{l}\text { High flavanol } \\
\text { cocoa/low flavanol } \\
\text { drink }\end{array}$ & $84 \mathrm{~d}(12 \mathrm{wk})$ & Control: 36 mg & 33.4 & & $\begin{array}{l}\text { Control: } 121 \text { (13.0)/ } \\
120.5 \text { (ng) }\end{array}$ & $\begin{array}{l}\text { Control: } 74 \text { (5.8)/73.8 } \\
\text { (ng) }\end{array}$ \\
\hline \multirow[t]{2}{*}{$\begin{array}{l}\text { Shiina et al. } \\
\text { [22]; Japan }\end{array}$} & Parallel ${ }^{a} ;$ & $45 \mathrm{~g} / \mathrm{d}$ & $\begin{array}{l}550 \mathrm{mg} \\
\text { polyphenols }\end{array}$ & $29.8 \mathrm{yr} ;$ & 20/19 & $\begin{array}{l}\text { Cocoa: } 116.4(12.7) / \\
121.0 \text { (12.7); }\end{array}$ & $\begin{array}{l}\text { Cocoa: } 64.7 \text { (11.7)/71.3 } \\
\text { (10.8); }\end{array}$ \\
\hline & $\begin{array}{l}\text { Dark/white } \\
\text { chocolate }\end{array}$ & $14 d$ & & 22.6 & & $\begin{array}{l}\text { Control: } 121.6(14.9) / \\
125.6 \text { (11.4) }\end{array}$ & $\begin{array}{l}\text { Control: } 72.2(13.8) / 77.4 \\
(11.6)\end{array}$ \\
\hline \multirow{2}{*}{$\begin{array}{l}\text { Ried et al. } \\
\text { (phase 1) [23]; } \\
\text { Australia }\end{array}$} & Parallel; & $50 \mathrm{~g} / \mathrm{d}$ & $\begin{array}{l}750 \mathrm{mg} \\
\text { polyphenols }\end{array}$ & $53.1 \mathrm{yr} ;$ & $11 / 10$ & $\begin{array}{l}\text { Cocoa: } 135.0(12.5) / \\
133.1(11.7)^{c}\end{array}$ & $\begin{array}{l}\text { Cocoa: } 83.6 \text { (10.6)/84.5 } \\
\text { (11.6); }\end{array}$ \\
\hline & $\begin{array}{l}\text { Dark chocolate/ } \\
\text { placebo pill }\end{array}$ & $56 \mathrm{~d}(8 w k)$ & & 26.6 & & $\begin{array}{l}\text { Control: } 135.7(12.4) / \\
130.8(18.3)^{c}\end{array}$ & $\begin{array}{l}\text { Control: } 77.8 \text { (8.6)/77.3 } \\
(10.0)\end{array}$ \\
\hline \multirow[t]{2}{*}{$\begin{array}{l}\text { Monagas et al. } \\
\text { [24]; Spain }\end{array}$} & Crossover; & $\begin{array}{l}40 \mathrm{~g} / \mathrm{d}+250 \\
\text { skim milk; } \\
\text { control: } 500 \mathrm{ml} \\
\text { skim milk }\end{array}$ & $\begin{array}{l}495 \mathrm{mg} \\
\text { polyphenols }\end{array}$ & $69.7 \mathrm{yr} ;$ & $42 / 42$ & $\begin{array}{l}\text { Cocoa: } 138(26) / 138 \\
\text { (16); }\end{array}$ & Cocoa: 84 (13)/82 (13); \\
\hline & $\begin{array}{l}\text { Cocoa powder in } \\
\text { milk/milk only }\end{array}$ & $28 d$ & & 27.6 & & $\begin{array}{l}\text { Control: } 138(26) / 135 \\
\text { (24) }\end{array}$ & Control: 84 (13)/81 (13) \\
\hline
\end{tabular}

${ }^{\text {a } 7-d a y ~ c o c o a / f l a v a n o l-f r e e ~ r u n-i n ~ p e r i o d . ~}$

b7-day washout period before crossover.

cMedian.

BMI, body mass index; DBP, diastolic blood pressure; g/d, grams per day; mg; milligrams; ml, millilitre; N, number of participants; ng, not given; SBP, systolic blood pressure; SD, standard deviation; yr, years; wk, weeks

Table 2 Characteristics of trials excluded from the meta-analysis

\begin{tabular}{|c|c|c|c|c|c|c|}
\hline Trial; location & $\begin{array}{l}\text { Study design; treatment/ } \\
\text { control groups }\end{array}$ & $\begin{array}{l}\text { Dosage; } \\
\text { duration }\end{array}$ & $\begin{array}{l}\text { Active ingredients } \\
\text { per daily dosage }\end{array}$ & $\begin{array}{l}\text { Mean age; } \\
\text { mean BMI }\end{array}$ & $\begin{array}{l}\mathrm{N} \\
\text { treatment/ } \\
\text { control }\end{array}$ & Reason for exclusion \\
\hline \multirow[t]{2}{*}{$\begin{array}{l}\text { Grassi et al. [19]; } \\
\text { Italy }\end{array}$} & Crossover $^{a, c}$ & $100 \mathrm{~g} / \mathrm{d}$ & 500 mg polyphenols & 33.9 yr; & $15 / 15$ & $\begin{array}{l}\text { Same population and protocol } \\
\text { as studied in Grassi et al. [11] }\end{array}$ \\
\hline & Dark/white chocolate & $15 d$ & & 22.6 & & \\
\hline \multirow{2}{*}{$\begin{array}{l}\text { Allen et al. [34], } \\
\text { Erdman et al. [35]; } \\
\text { USA }\end{array}$} & Crossover & $22 \mathrm{~g} / \mathrm{d}$ & Not reported & $44.7 \mathrm{yr}$ & $44 / 44$ & No cocoa-free control group \\
\hline & $\begin{array}{l}\text { Chocolate + plant sterols/ } \\
\text { chocolate + no plant } \\
\text { sterols }\end{array}$ & $28 \mathrm{~d}(4 \mathrm{wk})$ & & 27.8 & & \\
\hline \multirow{2}{*}{$\begin{array}{l}\text { Balzer et al. [36]; } \\
\text { USA }\end{array}$} & Parallel; & 3 drink mix/d & 963 mg flavanols; & $63.8 \mathrm{yr} ;$ & $21 / 20$ & No mean SBP/DBP (SD) reported \\
\hline & High/low-flavanol drink & $30 \mathrm{~d}(4 \mathrm{wk})$ & $\begin{array}{l}\text { control: } 75 \mathrm{mg} \\
\text { flavanols }\end{array}$ & 32.1 & & \\
\hline \multirow[t]{2}{*}{$\begin{array}{l}\text { Faridi et al. [37]; } \\
\text { USA }\end{array}$} & Crossover $^{\mathrm{a}, \mathrm{c}}$ & $\begin{array}{l}74 \mathrm{~g} \text { bar with } \\
22 \mathrm{~g} \text { cocoa/d; }\end{array}$ & $821 \mathrm{mg}$ flavanols & $53 \mathrm{yr}$ & $44 / 44$ & Duration $<2$ wk \\
\hline & $\begin{array}{l}\text { Dark chocolate/placebo } \\
\text { bar }\end{array}$ & $\begin{array}{l}\text { Single dose, } 1 \\
d\end{array}$ & & 30 & & \\
\hline \multirow[t]{2}{*}{$\begin{array}{l}\text { Ried et al. (phase 2) } \\
\text { [23]; Australia }\end{array}$} & Crossover $^{\mathrm{d}}$ & $\begin{array}{l}50 \mathrm{~g} / \mathrm{d} ; \\
\text { control: } 1 \\
\text { capsule/d; }\end{array}$ & $\begin{array}{l}750 \mathrm{mg} \\
\text { polyphenols; }\end{array}$ & $53.1 \mathrm{yr} ;$ & $26 / 26$ & $\begin{array}{l}\text { Crossover of two active } \\
\text { treatment groups, no true } \\
\text { control group }\end{array}$ \\
\hline & $\begin{array}{l}\text { Dark chocolate/Tomato } \\
\text { extract capsules }\end{array}$ & $\begin{array}{l}56 \text { days }(8 \\
\text { wks })\end{array}$ & $\begin{array}{l}\text { control: tomato } \\
\text { extract ( } 15 \mathrm{mg} \\
\text { lycopene) }\end{array}$ & 26.6 & & \\
\hline \multirow[t]{2}{*}{$\begin{array}{l}\text { Almoosawi et al. } \\
\text { [38]; UK }\end{array}$} & Crossover $^{\mathrm{a}, \mathrm{c}}$ & $20 \mathrm{~g} / \mathrm{d}$ & $\begin{array}{l}1000 \mathrm{mg} \\
\text { polyphenols; }\end{array}$ & $31 \mathrm{yr}$ & $14 / 14$ & $\begin{array}{l}\text { Two dosages, no true control } \\
\text { group }\end{array}$ \\
\hline & $\begin{array}{l}\text { Dark chocolate dosage 1/ } \\
\text { dosage } 2\end{array}$ & 14 days & control: 500 mg & 27.7 & & \\
\hline
\end{tabular}

7-day cocoa/flavanol-free run-in period.

${ }^{b} 2$-week run-in period.

${ }^{{ }^{7} 7-d a y ~ w a s h o u t ~ p e r i o d ~ b e f o r e ~ c r o s s o v e r . ~}$

d 4-week washout period.

For abbreviations, see Table 1 footnote. 
The 15 trial arms included in our meta-analysis were adequately randomised; double-blinding was achieved in five trial arms using low-flavanol-containing products as control [11,15,20,21]. All but three trial arms [15,22,24] assessed blood pressure as the primary outcome measure, and 12 of the 15 trial arms had a participant attrition of less than $20 \%$ [10,11,13,15-18,22-24] (Table 3).

Eight trial arms used a parallel study design [11,15-17,21-23], and seven study arms used a crossover design $[10,12,13,18,20,24]$. All but two [12,24] of the seven crossover trials incorporated a washout period of 7 days between the alternate treatment period. In eight trials the intervention period was 2 weeks (14 or 15 days) $[10-13,18,20,22]$, while longer intervention periods were trialled in seven studies (range 4-18 weeks) [15-17,21,23,24]. Eight trials employed a 7-day run-in period before commencing with the treatments [10,13,16-18,20,22] (Table 1).

Polyphenol content varied widely between the trials (range 30-1008 mg/day, Table 1). Although authors labelled the active ingredient in chocolate with a variety of terms (polyphenol, flavanol, proanthocyanidin, epicatechin and catechin), we are reasonably confident that the reported dosages of total polyphenol or measured derivates are comparable. There is some confusion in the literature about the correct labelling of the vasoactive chemical components in cocoa, and terms are often used interchangeably and sometimes incorrectly.

Table 3 Quality assessment of trials included in the meta-analysis

\begin{tabular}{|c|c|c|c|c|c|c|}
\hline Trial ID & $\begin{array}{l}\text { Total } \\
\text { score }\end{array}$ & Blinding & $\begin{array}{l}\text { Outcome measure: } \\
\text { blood pressure }\end{array}$ & $\begin{array}{l}\text { Loss to } \\
\text { follow-up }\end{array}$ & Funding source & $\begin{array}{l}\text { Compliance; other } \\
\text { chocolate diary }\end{array}$ \\
\hline \multirow[t]{3}{*}{$\begin{array}{l}\text { Scores } \\
\text { given }\end{array}$} & & 1: choc + control blinded & 1: Primary & $1:<20 \%$ & $\begin{array}{l}\text { 1: Sponsor not involved in } \\
\text { data collection, analysis }\end{array}$ & $\begin{array}{l}\text { 1: Compl assessed; no sign } \\
\text { diff between groups }\end{array}$ \\
\hline & & 0.5: control blinded & 0.5: Secondary & $0: \geq 20 \%$ & 0: Sponsor involved & $\begin{array}{l}\text { 1: Compl assessed, sign diff } \\
\text { included in analysis }\end{array}$ \\
\hline & & 0: No blinding & & & & 0: Compl not assessed \\
\hline $\begin{array}{l}\text { Taubert } \\
\text { et al. [10] }\end{array}$ & 4 & 0: dark/white & 1 & 1: none & 1 & 1 \\
\hline $\begin{array}{l}\text { Murphy } \\
\text { et al. [15] }\end{array}$ & 3.5 & 1: cocoa tablets & 0.5 & $\begin{array}{l}1: 9.5 \%(4 \\
\text { of } 42)\end{array}$ & 0: supported by Mars & $\begin{array}{l}\text { 1: tablet count, } 7 \text { day } \\
\text { weight food records } \times 2\end{array}$ \\
\hline $\begin{array}{l}\text { Engler et al. } \\
\text { [11] }\end{array}$ & 5 & 1: flav bar matched & 1 & 1: none & 1 & 1 \\
\hline $\begin{array}{l}\text { Fraga et al. } \\
{[12]}\end{array}$ & 2.5 & 0: dark/white & 1 & $\begin{array}{l}1: 3.6 \%(1 \\
\text { of } 28)\end{array}$ & $0: 3$ authors from Mars & $\begin{array}{l}0.5 \text { : reported what } \\
\text { consumed, no diary }\end{array}$ \\
\hline $\begin{array}{l}\text { Grassi et al. } \\
\text { [13] }\end{array}$ & 3.5 & 0: dark/white & 1 & 1: none & 0.5: unclear & 1: daily diary \\
\hline $\begin{array}{l}\text { Grassi et al. } \\
\text { [13] }\end{array}$ & 3.5 & 0: dark/white & 1 & 1: none & 0.5: unclear & 1: daily diary \\
\hline $\begin{array}{l}\text { Taubert et } \\
\text { al. [16] }\end{array}$ & 4 & 0: dark/white & 1 & 1: none & 1 & 1: food diary \\
\hline $\begin{array}{l}\text { Crews et al. } \\
{[17]}\end{array}$ & 2.5 & $\begin{array}{l}0.5 \text { : flav drink; }>50 \% \text { assumed } \\
\text { correct group they were in }\end{array}$ & 1 & $\begin{array}{l}0.5: 11 \% \\
(11 \text { of } 101)\end{array}$ & $\begin{array}{l}0.5 \text { : independent industry } \\
\text { research grant, supplier }\end{array}$ & 0: not reported \\
\hline $\begin{array}{l}\text { Grassi et al. } \\
{[18]}\end{array}$ & 3.5 & 0: dark/white & 1 & 1: none & 0.5: unclear & 1: diary \\
\hline $\begin{array}{l}\text { Muniyappa } \\
\text { et al. [20] }\end{array}$ & 3 & $\begin{array}{l}\text { 1: flav drink; blinding assessed } \\
\text { ok }\end{array}$ & 1 & $\begin{array}{l}0: 31 \%(9 \\
\text { of } 29)\end{array}$ & 1 & $0:$ not reported \\
\hline $\begin{array}{l}\text { Davison } \\
\text { et al. [21] }\end{array}$ & 3 & 1: flav drink & 1 & $\begin{array}{l}0: 21 \%(14 \\
\text { of } 65)\end{array}$ & 0: Mars Financial support & $\begin{array}{l}\text { 1: diet + background } \\
\text { exercise }\end{array}$ \\
\hline $\begin{array}{l}\text { Davison } \\
\text { et al. [21] }\end{array}$ & 3 & 1: flav drink & 1 & $\begin{array}{l}0: 21 \%(14 \\
\text { of } 65)\end{array}$ & 0: Mars Financial support & $\begin{array}{l}\text { 1: diet }+ \text { background } \\
\text { exercise }\end{array}$ \\
\hline $\begin{array}{l}\text { Shiina et al. } \\
{[22]}\end{array}$ & 2 & 0: dark/white & 0 & 1: none & 1 & 0 \\
\hline $\begin{array}{l}\text { Ried et al. } \\
{[23]}\end{array}$ & 4.5 & 0.5: control blinded & 1 & $\begin{array}{l}1: 8 \%(3 \text { of } \\
39)\end{array}$ & 1 & 1: diary \\
\hline $\begin{array}{l}\text { Monagas } \\
\text { et al. [24] }\end{array}$ & 3.5 & 0: cocoa powder in milk/milk & 0.5 & 1: none & 1 & $\begin{array}{l}\text { 1: diet monitoring (3-day } \\
\text { food questionnaires } \times 3 \text { ) }\end{array}$ \\
\hline
\end{tabular}

All 15 trial arms were adequately randomised.

Choc, chocolate; compl, compliance; flav, flavanol; sign diff, significant difference; 


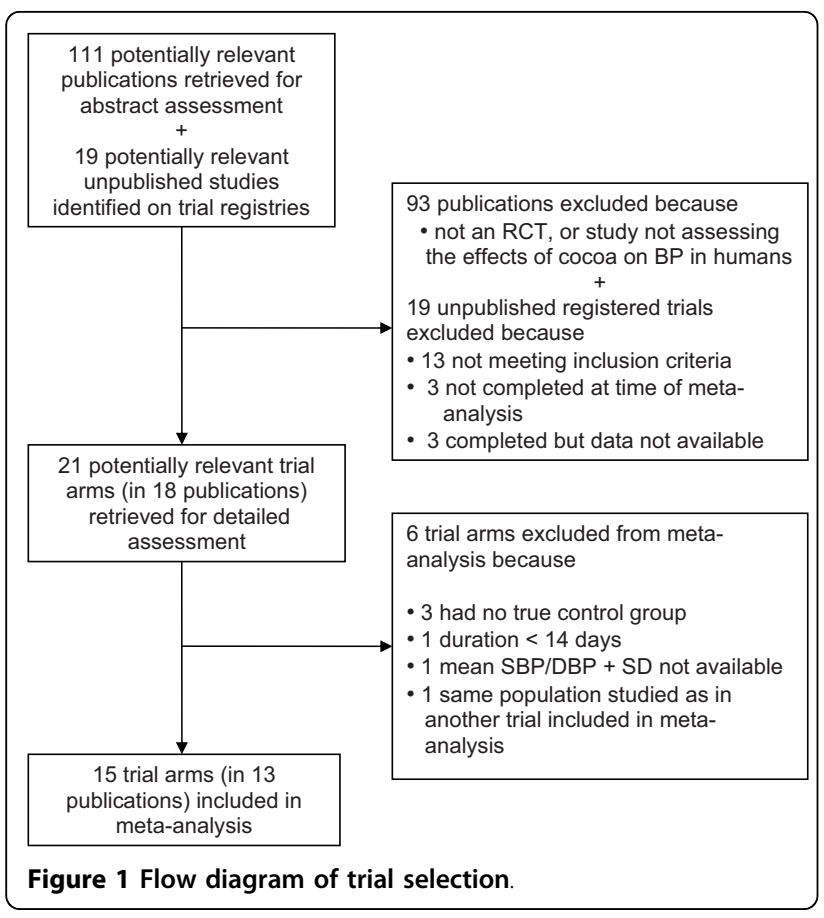

Furthermore, the most commonly used methods for polyphenol content analysis (high performance liquid chromatography [39] and Folin-Ciocalteu method [40]) each measure both monomer (epicatechin and catechin) and oligomer (proanthocyanidin) polyphenol components. These polyphenol components belong to the flavanols or flavanoids, which make up $99 \%$ of all polyphenols in cocoa [41,42]. However, we were not able to compare findings by method of polyphenol content analysis, as details were not available for all trials.

We collated information on age and weight/BMI because age and BMI may influence responsiveness to blood pressure treatment $[43,44]$. Mean age and BMI varied substantially between trials: mean age ranged between 18 and 70 years, and mean BMI was in the overweight/obese category for 9 of the 15 trials (mean BMI: <25, $\mathrm{n}=6$; 25-30, $\mathrm{n}=6$; $>30, \mathrm{n}=3$ ).

\section{Meta-analysis}

Meta-analysis of all 15 trial arms revealed a significant blood pressure-reducing effect of cocoa/chocolate compared with control (pooled mean SBP: -3.16 [95\% CI, -5.08, -1.23] $\mathrm{mmHg}, P=0.001$; pooled mean DBP: -2.02 [95\% CI, $-3.35,-0.69] \mathrm{mmHg}, P=0.003$ ) (Figure 2). Heterogeneity between trials was high (SBP: $I^{2}=74 \%$; DBP: $I^{2}=62 \%$ ), prompting subgroup meta-analysis by baseline blood pressure as well as meta-regression and sensitivity analyses.

We pooled trial arms with mean baseline SBP in the hypertensive range $(\mathrm{SBP} \geq 140 \mathrm{mmHg})$ and trial arms with mean baseline SBP of $<140 \mathrm{~mm} \mathrm{Hg}$. While metaanalysis of the SBP hypertensive subgroup remained significant ( $\mathrm{SBP}_{\text {hyper: }}$-5.02 [95\% CI, -7.99, -2.05] $\mathrm{mmHg}$; $P=0.0009$; Figure 3A), meta-analysis of the SBP normotensive subgroup demonstrated no significant difference in blood pressure reduction between the chocolate/ cocoa group and the control group $\left(\mathrm{SBP}_{\text {normo: }}:-1.56\right.$ [95\% CI, -3.81, 0.68] mmHg, $P=0.17$; Figure 3B). Heterogeneity remained high in the hypertensive subgroup, but was reduced in the SBP normotensive subgroup (SBP hyper: $I^{2}=90 \%$; $\mathrm{SBP}_{\text {normo }}: I^{2}=23 \%$ ).

For subgroup analysis of diastolic blood pressure, we pooled trial arms with mean baseline DBP in the hypertensive and prehypertensive range (DBP $\geq 80 \mathrm{mmHg}$ ) and trials with mean baseline DBP $<80 \mathrm{~mm} \mathrm{Hg}$. Three of the six trial arms in the subgroup with $\mathrm{DBP} \geq 80$ $\mathrm{mmHg}$ reported mean DBP values at baseline in the hypertensive range $(\geq 90 \mathrm{mmHg})[13,18,20]$, while three trials reported mean DBP at baseline in the prehypertensive range $(84-86 \mathrm{mmHg})[10,16,24]$.

Results of DBP subgroup analyses were similar to the results of SBP subgroup analyses. While the DBP pre-/ hypertensive subgroup analysis remained significant (DBP hyper: -2.73 [95\% CI, -4.89, -0.58] mmHg, $P=0.01$; $I^{2}=79 \%$; Figure 3C), DBP normotensive subgroup analysis demonstrated no significant difference between chocolate and control groups (DBP ${ }_{\text {normo: }}:-1.28$ [95\% CI, -2.88, 0.33] mmHg, $P=0.12 ; I^{2}=24 \%$; Figure 3D).

Funnel plots and Egger's test indicated no publication bias (Figure 4).

\section{Meta-regression and sensitivity analysis}

Five variables tested (dosage, duration, quality score, age and BMI) did not show any significant association with blood pressure outcomes in the meta-regression analysis, while study design (parallel versus crossover) was borderline significantly associated with BP outcome (SBP: $P=0.053$; DBP: $P=0.060$ ); and type of control (flavanol-free control versus low-flavanol product as control) was borderline significant for DBP $(P=0.052)$.

Subgroup analyses revealed reduced heterogeneity in the subgroup including parallel trials, but high heterogeneity remained in the subgroup with crossover trials (Table 4). Similarly, the subgroup including low-flavanol products as control demonstrated lower heterogeneity and no significant difference between treatment groups, in contrast to the subgroup with flavanol-free unblinded controls (Table 4).

However, the results of these subgroup analyses need to be interpreted cautiously, as three of seven trial arms with a crossover study design were conducted by the same study team $[13,18]$, and five of nine study arms using a flavanol-free control were conducted by two teams within similar population groups $[10,13,16,18]$. 


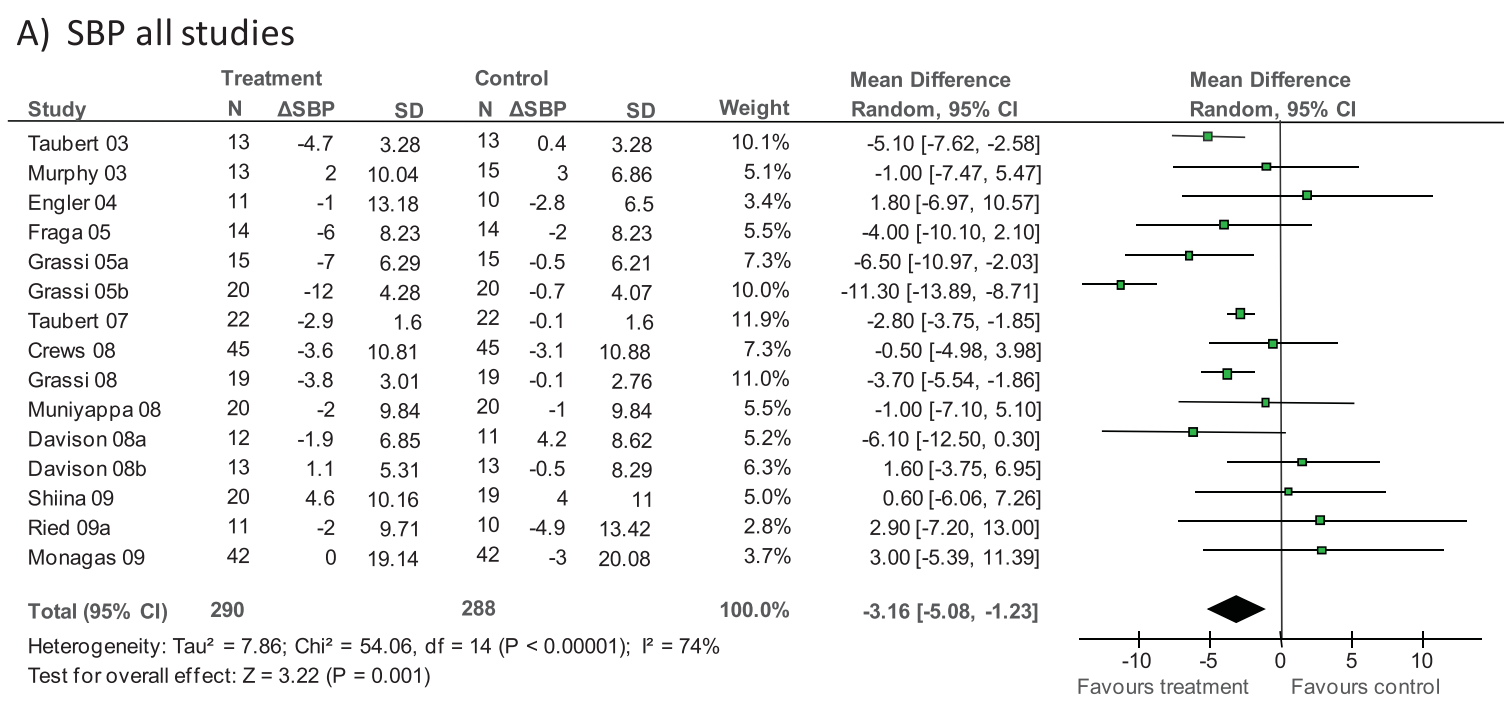

\section{B) DBP all studies}

\begin{tabular}{|c|c|c|c|c|c|c|c|c|c|c|c|}
\hline Study & \multicolumn{2}{|c|}{ Treatment } & SD & \multicolumn{2}{|c|}{ Control } & SD & Weight & $\begin{array}{l}\text { Mean Difference } \\
\text { Random, } 95 \% \mathrm{Cl}\end{array}$ & \multicolumn{3}{|c|}{$\begin{array}{l}\text { Mean Difference } \\
\text { Random, } 95 \% \mathrm{Cl}\end{array}$} \\
\hline Taubert 03 & 13 & -1.6 & 3.68 & 13 & 0.3 & 3.4 & $8.7 \%$ & $-1.90[-4.62,0.82]$ & & & \\
\hline Murphy 03 & 13 & 1 & 8.99 & 15 & 0 & 6.07 & $3.8 \%$ & $1.00[-4.77,6.77]$ & & & \\
\hline Engler 04 & 11 & 0.9 & 6.16 & 10 & -0.1 & 4.76 & $5.1 \%$ & $1.00[-3.69,5.69]$ & & & \\
\hline Fraga 05 & 14 & -5 & 5.99 & 14 & -1 & 5.99 & $5.4 \%$ & $-4.00[-8.44,0.44]$ & & & \\
\hline Grassi 05a & 15 & -4.2 & 4.23 & 15 & -0.3 & 4.32 & $7.9 \%$ & $-3.90[-6.96,-0.84]$ & & & \\
\hline Grassi 05b & 20 & -7.8 & 4.11 & 20 & -0.2 & 3.76 & $9.3 \%$ & $-7.60[-10.04,-5.16]$ & & & \\
\hline Taubert 07 & 22 & -1.9 & 1 & 22 & 0 & 1.8 & $12.9 \%$ & $-1.90[-2.76,-1.04]$ & $\rightarrow$ & & \\
\hline Crews 08 & 45 & -0.5 & 6.31 & 45 & -0.6 & 6.29 & $9.0 \%$ & $0.10[-2.50,2.70]$ & & & \\
\hline Grassi 08 & 19 & -3.9 & 3.54 & 19 & -0.2 & 2.89 & $10.3 \%$ & $-3.70[-5.75,-1.65]$ & & & \\
\hline Muniyappa 08 & 20 & -3 & 9.84 & 20 & -4 & 9.84 & $3.5 \%$ & $1.00[-5.10,7.10]$ & & & \\
\hline Davison $08 a$ & 12 & -1.8 & 5.2 & 11 & 2.8 & 5.64 & $5.4 \%$ & $-4.60[-9.05,-0.15]$ & & & \\
\hline Davison 08b & 13 & -0.5 & 3.61 & 13 & -0.2 & 5.41 & $6.9 \%$ & $-0.30[-3.84,3.24]$ & & & \\
\hline Shiina 09 & 20 & 6.6 & 9.04 & 19 & 5.2 & 10.36 & $3.5 \%$ & $1.40[-4.72,7.52]$ & & $\square$ & \\
\hline Ried 09a & 11 & 0.9 & 8.93 & 10 & -0.5 & 7.55 & $2.8 \%$ & $1.40[-5.65,8.45]$ & & $\rightarrow$ & \\
\hline Monagas 09 & 42 & -2 & 10.4 & 42 & -3 & 10.4 & $5.4 \%$ & $1.00[-3.45,5.45]$ & & & \\
\hline Total $(95 \% \mathrm{Cl})$ & 290 & & & 288 & & & $100.0 \%$ & $-2.02[-3.35,-0.69]$ & & & \\
\hline \multicolumn{8}{|c|}{$\begin{array}{l}\text { Heterogeneity: } \mathrm{Tau}^{2}=3.40 ; \mathrm{Chi}^{2}=36.75, \mathrm{~d} \\
\text { Test for overall effect: } \mathrm{Z}=2.97(P=0.003)\end{array}$} & & $\begin{array}{ll}10 & -5 \\
-10 & \\
\text { Favours treatment }\end{array}$ & 0 Favours & $\begin{array}{ll}1 & 1 \\
5 & 10 \\
\text { rs control }\end{array}$ \\
\hline
\end{tabular}

Figure 2 Meta-analysis of the effect of chocolate/cocoa on (A) systolic blood pressure or (B) diastolic blood pressure. $N$, number of participants; $\triangle \mathrm{SBP} / \triangle \mathrm{DBP}$, difference in mean SBP/DBP between start and end of intervention; SD, standard deviation; Cl, confidence interval

Therefore, study design or type of control might in fact be indirect predictors of BP outcome through other factors such as dietary habits of the study population.

Sensitivity analyses excluding trial [16], as this trial used a notably lower dose and longer duration than the other trials, did not change the results appreciably, with one exception: A small but statistically significant difference between treatment groups in subgroup analyses of trials with a parallel design shifted to a nonsignificant effect when trial [16] was excluded (Table 4).

\section{Discussion}

Our meta-analysis including 15 trial arms demonstrated a small but significant blood pressure-reducing effect of flavanol-rich cocoa products compared with control (mean BP change \pm SE: SBP: $-3.2 \pm 1.9 \mathrm{mmHg}, P=$ 0.001; DBP: $-2.0 \pm 1.3 \mathrm{mmHg}, P=0.003$ ). These effect sizes were smaller than in previous meta-analyses including fewer trials (Taubert et al. (2007), 5 trials, SBP: $-4.7 \pm 2.9 \mathrm{mmHg}, P=0.002$; DBP: $-2.8 \pm 2.0$ $\mathrm{mmHg}, P=0.006$, [9]; and Desch et al. (2010), 10 trials, SBP: $-4.5 \pm 1.4 \mathrm{mmHg}, P=0.001$, DBP: $-2.5 \pm 1.4$ $\mathrm{mmHg}, P=0.001,[14])$.

In contrast to previous meta-analyses [9,14], subgroup analyses in our larger meta-analysis suggested that there is a difference in outcome dependent on baseline blood pressure (hypertensive versus normotensive). While meta-analyses of the hypertensive subgroups found 
A) SBP hypertensive subgroup ( $\geq 140 \mathrm{~mm} \mathrm{Hg}$ )

\begin{tabular}{|c|c|c|c|c|c|c|c|c|c|c|c|c|}
\hline \multirow[b]{2}{*}{ Study } & \multicolumn{3}{|c|}{ Treatment } & \multicolumn{3}{|c|}{ Control } & \multirow[b]{2}{*}{ Weight } & \multirow{2}{*}{$\begin{array}{l}\text { Mean Difference } \\
\text { Random, } 95 \% \mathrm{Cl}\end{array}$} & \multirow{2}{*}{\multicolumn{4}{|c|}{$\begin{array}{l}\text { Mean Difference } \\
\text { Random, } 95 \% \mathrm{Cl}\end{array}$}} \\
\hline & $\mathrm{N}$ & $\triangle \mathrm{SBP}$ & SD & $\mathrm{N}$ & $\triangle \mathrm{SBP}$ & SD & & & & & & \\
\hline Taubert 03 & 13 & -4.7 & 3.28 & 13 & 0.4 & 3.28 & $20.9 \%$ & $-5.10[-7.62,-2.58]$ & & & & \\
\hline Grassi 05b & 20 & -12 & 4.28 & 20 & -0.7 & 4.07 & $20.7 \%$ & $-11.30[-13.89,-8.71]$ & $\longrightarrow$ & & & \\
\hline Taubert 07 & 22 & -2.9 & 1.6 & 22 & -0.1 & 1.6 & $23.9 \%$ & $-2.80[-3.75,-1.85]$ & & $\rightarrow-$ & & \\
\hline Grassi 08 & 19 & -3.8 & 3.01 & 19 & -0.1 & 2.76 & $22.4 \%$ & $-3.70[-5.54,-1.86]$ & & $\longrightarrow$ & & \\
\hline Muniyappa 08 & 20 & -2 & 9.84 & 20 & -1 & 9.84 & $12.1 \%$ & $-1.00[-7.10,5.10]$ & & & & \\
\hline Total $(95 \% \mathrm{Cl})$ & 94 & & & 94 & & & $100.0 \%$ & $-5.02[-7.99,-2.05]$ & & & & \\
\hline $\begin{array}{l}\text { Heterogeneity: } \\
\text { Test for overall }\end{array}$ & $=9.36$ & $\begin{array}{l}; \mathrm{Chi}^{2}= \\
\text { 3.31(P }\end{array}$ & $\begin{array}{l}=38.37 \\
=0.00\end{array}$ & $=4($ & $b<0.00$ & 0001); । & $90 \%$ & & $\begin{array}{l}-10 \\
\text { Favours } t\end{array}$ & $\begin{array}{c}-5 \\
\text { reatment }\end{array}$ & $\begin{array}{lr}0 & 5 \\
& \text { Favours }\end{array}$ & $\begin{array}{c}10 \\
\text { ontrol }\end{array}$ \\
\hline
\end{tabular}

\section{B) SBP normotensive subgroup ( $<140 \mathrm{~mm} \mathrm{Hg}$ )}

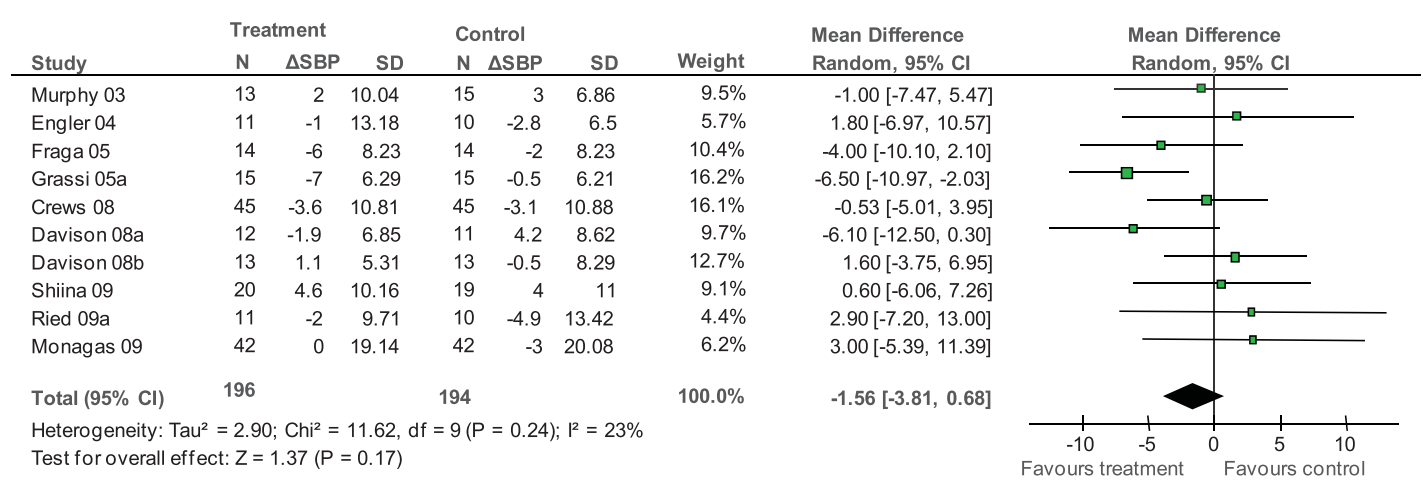

C) DBP (pre-) hypertensive subgroup ( $\geq 80 \mathrm{~mm} \mathrm{Hg}$ )

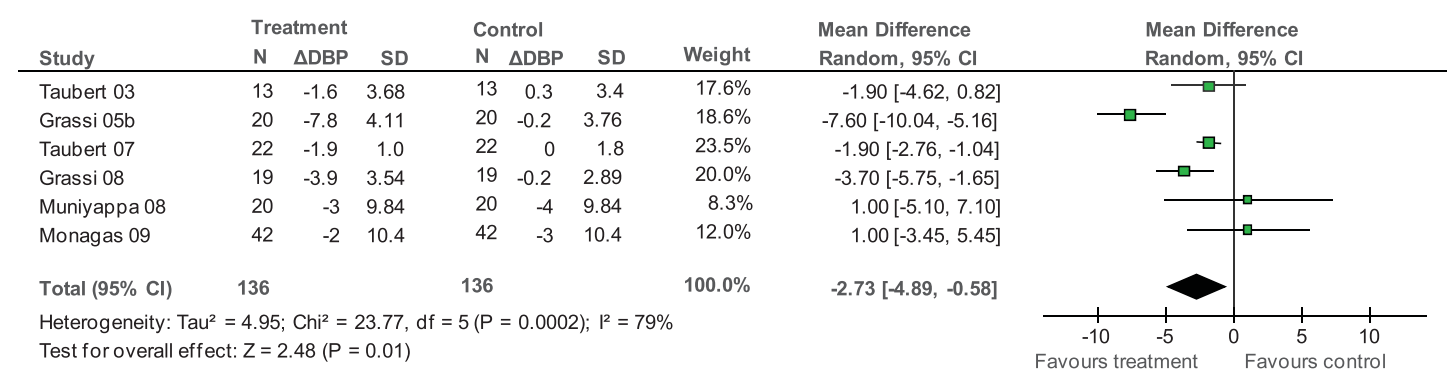

\section{D) DBP normotensive subgroup ( $<80 \mathrm{~mm} \mathrm{Hg}$ )}

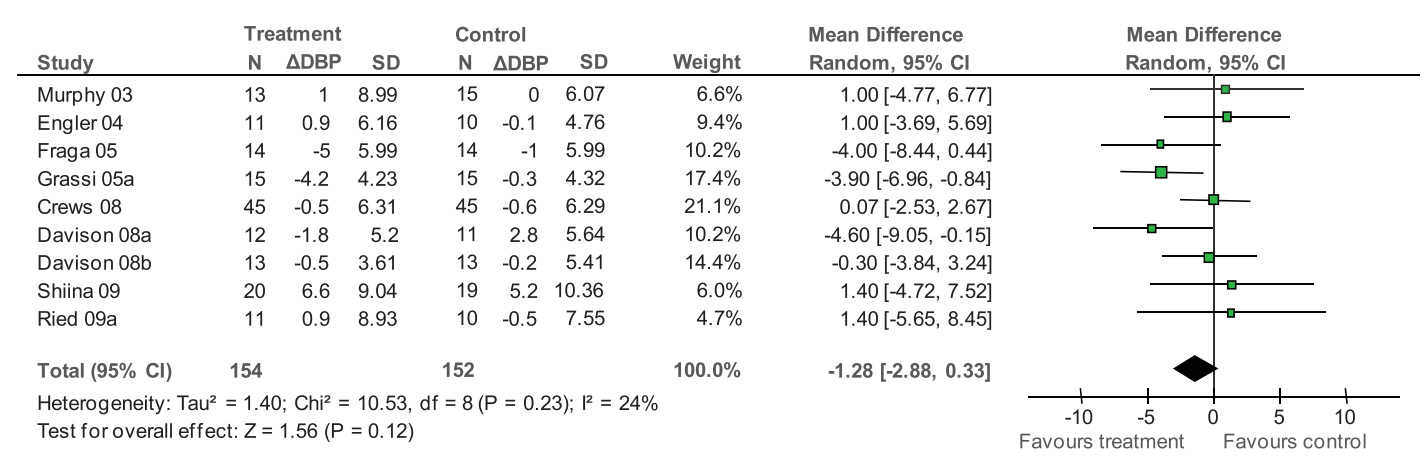

Figure 3 Subgroup meta-analysis of the effect of chocolate/cocoa on (A) systolic blood pressure of hypertensive subjects ( $\geq 140$ $\mathrm{mmHg}$ at baseline) or (B) 'normotensive' subjects ( $140 \mathrm{mmHg}$ at baseline) and on (C) diastolic blood pressure of (pre-)hypertensive subjects ( $\geq 80 \mathrm{mmHg}$ ) or (D) 'normotensive' subjects $(<80 \mathrm{mmHg}$ ). See Figure 2 legend for abbreviation definitions. 


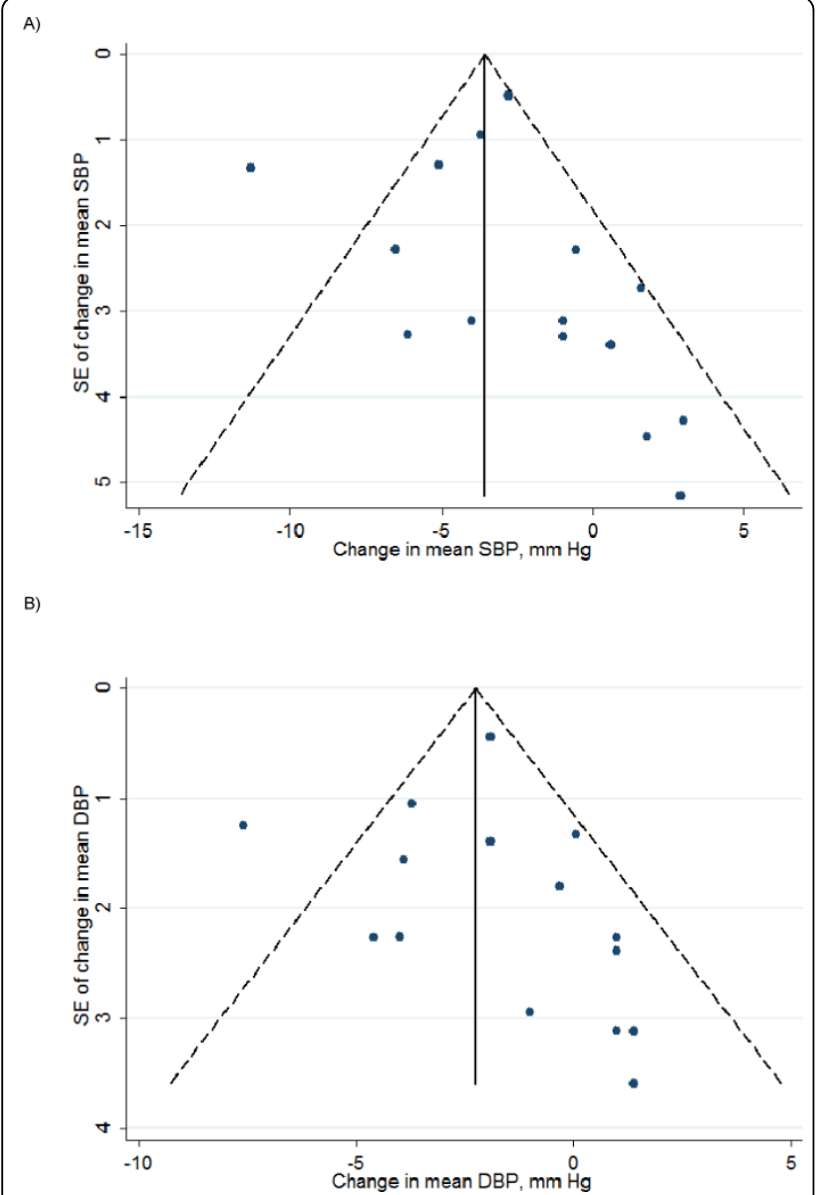

Figure 4 Funnel plots of trials included in the meta-analysis for (A) systolic blood pressure and (B) diastolic blood pressure. The vertical line of Begg's funnel plot represents the pooled mean effect size, and the dotted lines represent the $95 \%$ confidence interval. $P$ values are derived from Egger's test.

significant reductions $(\mathrm{SBP}:-5.0 \pm 3.0 \mathrm{mmHg}, P=$ 0.0009; DBP: $-2.7 \pm 2.2 \mathrm{mmHg}, P=0.01$ ), analyses of normotensive subgroups did not demonstrate a significant reduction in blood pressure of flavanol-rich cocoa products (SBP: $-1.6 \pm 2.3 \mathrm{mmHg}, P=0.17$; DBP: $-1.3 \pm$ $1.6 \mathrm{mmHg}, P=0.12$ ). These findings are in line with meta-analyses of other nutritional supplements on blood pressure, which similarly found that blood pressure was significantly reduced in hypertensive subgroups but not in the normotensive subgroups $[30,45]$.

Heterogeneity was reduced satisfactorily in the normotensive subgroups, indicating that trials in these subgroup analyses are highly comparable and the metaanalyses results can be interpreted with confidence. In contrast, heterogeneity remained high in the hypertensive subgroups, influenced greatly by one relatively small study arm [13], which demonstrated a large blood pressure reduction not matched by the other trials.
Therefore, effect sizes and levels of significance of the subgroup meta-analyses of trials with (pre-)hypertensive subjects at baseline should be interpreted more cautiously.

The relatively modest but significant blood pressurelowering effect of cocoa in the hypertensive subgroup is clinically relevant: a decline of $5 \mathrm{mmHg}$ in systolic blood pressure may reduce the risk of a cardiovascular event by about $20 \%$ over 5 years [46]. Furthermore, the effect of cocoa in a hypertensive population is comparable to other lifestyle modifications, such as moderate physical activity $(30 \mathrm{~min} / \mathrm{d})$ may reduce SBP by 4 $9 \mathrm{mmHg}$ [5].

Meta-regression analyses suggested study design (parallel versus crossover) and type of control (flavanol-free versus low-flavanol) to be significant predictors of blood pressure outcome but failed to show any statistically significant associations in the other variables tested. However, study design as well as type of control might be indirect predictors, as about half the trials using a crossover design and white chocolate as flavanol-free control were conducted by the same two teams and within similar study populations $[10,13,16,18]$. It is possible that participants shared characteristics that contributed to their responsiveness to cocoa products, such as local dietary habits or genetic/ethnic disparity $[47,48]$. Inclusion of trial location as a variable was impractical in our meta-regression analysis; however, future research may explore this further.

Furthermore, results of trials using flavanol-free controls, including white chocolate or milk, might overestimate the effect of the active treatment, owing to potential bias of unblinded participants. Therefore, related subgroup analyses need to be interpreted cautiously.

Meta-regression analysis did not suggest an association between dosage, duration, quality of trials, age, BMI and blood pressure outcome. However, inclusion of future trials in meta-regression analysis might provide further insight into predicting factors.

While regular consumption of flavanol-rich cocoa products may have a beneficial short-term effect in reducing blood pressure in hypertensive individuals, the practicability of chocolate or cocoa drinks as long-term treatment is questionable. A recent small study by our team investigating the acceptability of commercially available chocolate bars as an alternative treatment to capsules concluded that daily chocolate consumption for blood pressure may not be an acceptable and practical treatment option [23].

\section{Conclusion}

Our meta-analysis of 15 trial arms suggests that dark chocolate and flavanol-rich cocoa products are 
Table 4 Subgroup analyses by study design and type of control

\begin{tabular}{|c|c|c|c|c|c|c|c|}
\hline \multirow[t]{2}{*}{ Subgroups } & & \multicolumn{3}{|l|}{ SBP } & \multicolumn{3}{|l|}{ DBP } \\
\hline & & $\begin{array}{l}\text { Mean difference in } \mathrm{mmHg} \\
(95 \% \mathrm{Cl})\end{array}$ & $P$ & $I^{2}$ & $\begin{array}{l}\text { Mean difference in } \mathrm{mmHg} \\
(95 \% \mathrm{Cl})\end{array}$ & $P$ & $I^{2}$ \\
\hline \multirow{3}{*}{$\begin{array}{l}\text { Study } \\
\text { design }\end{array}$} & Crossover $[10,12,13,18,20,21,23]$ & $-4.91(-7.94,-1.88)$ & 0.002 & $80 \%$ & $-3.24(-5.36,-1.12)$ & 0.003 & $67 \%$ \\
\hline & Parallel [11,15-17,21-23] & $-2.01(-3.42,-0.60)$ & 0.005 & $9 \%$ & $-1.20(-2.29,-0.11)$ & 0.03 & $12 \%$ \\
\hline & Parallel $^{\mathrm{a}}[11,15,17,21-23]$ & $-0.43(-2.80,1.94)$ & 0.72 & $0 \%$ & $-0.25(-1.83,1.33)$ & 0.75 & $0 \%$ \\
\hline \multirow[t]{2}{*}{$\begin{array}{l}\text { Type of } \\
\text { control }\end{array}$} & $\begin{array}{l}\text { Flavanol-free: white chocolate, milk, pills } \\
{[11,15,17,20,21]}\end{array}$ & $-4.21(-6.65,-1.77)$ & 0.0007 & $82 \%$ & $-2.81(-4.52,-1.10)$ & 0.001 & $70 \%$ \\
\hline & $\begin{array}{l}\text { Low-flavanol: drink, bar, tablet } \\
{[10,13,16,18,22-24]}\end{array}$ & $0.74(-2.92,4.40)$ & 0.69 & $53 \%$ & $-0.38(-2.00,1.24)$ & 0.65 & $0 \%$ \\
\hline
\end{tabular}

SBP, systolic blood pressure; DBP, diastolic blood pressure; $\mathrm{mmHg}$, millimetre mercury; $\mathrm{Cl}$, confidence interval; $P$, probability value $(P<0.05$ statistically significant); $l^{2}$, heterogeneity test.

aSensitivity analysis excluding trial [16], as this trial used a notably lower dose and longer duration than the other trials included in the meta-analysis.

superior to placebo in reducing systolic hypertension and diastolic prehypertension. However, flavanol-rich cocoa products did not significantly reduce mean blood pressure below $140 \mathrm{mmHg}$ systolic or $80 \mathrm{mmHg}$ diastolic. Additional trials of hypertensive populations are needed to elucidate whether local dietary habits or genetic factors influence the blood pressure-lowering effect of cocoa.

\section{List of Abbreviations}

BP: blood pressure; BMI: body mass index; CI: confidence interval; DBP: diastolic blood pressure; mg: milligrams; mm Hg: millimetre mercury; RCT: randomised controlled trial; SBP: systolic blood pressure; SD: standard deviation.

\section{Acknowledgements}

We gratefully acknowledge the assistance by N Funabashi [22] and D Grassi [18] who provided unpublished data for inclusion in our meta-analysis. This study was supported by the Royal Australian College of General Practitioners (RACGP) 2006 Cardiovascular Health Research Grant, and the Australian Government Primary Health Care Research Evaluation Development (PHCRED) Program.

\section{Author details}

'Discipline of General Practice, The University of Adelaide, Adelaide, SA 5005, Australia. ${ }^{2}$ Discipline of Public Health, The University of Adelaide, Adelaide, SA 5005, Australia.

\section{Authors' contributions}

KR, ORF and NPS conceptualised the study and obtained funding. Data was acquired independently by KR and PF. KR and TS undertook data analysis and interpretation. KR prepared the manuscript with contributions from all co-authors. All authors approved the final version.

\section{Competing interests}

The authors declare that they have no competing interests.

Received: 4 June 2010 Accepted: 28 June 2010 Published: 28 June 2010

\section{References}

1. Corti R, Flammer AJ, Hollenberg NK, Lusche TF: Cocoa and cardiovascular health. Circulation 2009, 119:1433-1441.
2. Selmi C, Cocchi CA, Lanfredini M, Keen CL, Gershwin ME: Chocolate at heart: the anti-inflammatory impact of cocoa flavanols. Mol Nutr Food Res 2008, 52:1340-1348.

3. Lewington S, Clarke R, Qizilbash N, Peto R, Collins R: Age-specific relevance of usual blood pressure to vascular mortality: a meta-analysis of individual data for one million adults in 61 prospective studies. Lancet 2002, 360:1903-1913

4. Mclnnes GT: Lowering blood pressure for cardiovascular risk reduction. J Hypertens Suppl 2005, 23:S3-S8.

5. Chobanian AV, Bakris GL, Black HR, Cushman WC, Green LA, Izzo JL Jr, Jones DW, Materson BJ, Oparil S, Wright JT Jr, Roccella EJ, NationalHeart, Lung, and Blood Institute Joint National Committee on Prevention, Detection, Evaluation, and Treatment of High Blood Pressure; National High Blood Pressure Education Program Coordinating Committee: The Seventh Report of the Joint National Committee on Prevention, Detection, Evaluation, and Treatment of High Blood Pressure: the JNC 7 report. JAMA 2003, 289:2560-2572.

6. Fisher ND, Hollenberg NK: Aging and vascular responses to flavanol-rich cocoa. J Hypertens 2006, 24:1575-1580.

7. Fisher ND, Hughes M, Gerhard-Herman M, Hollenberg NK: Flavanol-rich cocoa induces nitric-oxide-dependent vasodilation in healthy humans. J Hypertens 2003, 21:2281-2286.

8. Karim M, McCormick K, Kappagoda CT: Effects of cocoa extracts on endothelium-dependent relaxation. J Nutr 2000, 130(8S Suppl):2105S-2108S.

9. Taubert D, Roesen R, Schomig E: Effect of cocoa and tea intake on blood pressure: a meta-analysis. Arch Intern Med 2007, 167:626-634.

10. Taubert D, Berkels R, Roesen R, Klaus W: Chocolate and Blood Pressure in Elderly Individuals with Isolated Systolic Hypertension. JAMA 2003, 290:1029-1030.

11. Engler MB, Engler MM, Chen CY, Malloy MJ, Browne A, Chiu EY, Kwak H-K, Milbury P, Paul SM, Blumberg J, Mietus-Snyder ML: Flavonoid-Rich Dark Chocolate Improves Endothelial Function and Increases Plasma Epicatechin Concentrations in Healthy Adults. J Am Coll Nutr 2004, 23:197-204.

12. Fraga CG, Actis-Goretta L, Ottaviani JI, Carrasquedo F, Lotito SB, Lazarus S, Schmitz HH, Keen CL: Regular consumption of a flavanol-rich chocolate can improve oxidant stress in young soccer players. Clin Dev Immunol 2005, 12:11-17.

13. Grassi D, Necozione S, Lippi C, Croce G, Valeri L, Pasqualetti P, Desideri G, Blumberg JB, Ferri $C$ : Cocoa reduces blood pressure and insulin resistance and improves endothelium-dependent vasodilation in hypertensives. Hypertension 2005, 46:398-405.

14. Desch S, Schmidt J, Kobler D, Sonnabend M, Eitel I, Sareban M, Rahimi K, Schuler G, Thiele H: Effect of cocoa products on blood pressure: systematic review and meta-analysis. Am J Hypertens 2010, 23:97-103.

15. Murphy KJ, Chronopoulos AK, Singh I, Francis MA, Moriarty H, Pike MJ, Turner AH, Mann NJ, Sinclair AJ: Dietary flavanols and procyanidin oligomers from cocoa (Theobroma cacao) inhibit platelet function. Am J Clin Nutr 2003, 77:1466-1473. 
16. Taubert D, Roesen R, Lehmann C, Jung N, Schomig E: Effects of low habitual cocoa intake on blood pressure and bioactive nitric oxide: a randomized controlled trial. JAMA 2007, 298:49-60.

17. Crews WD Jr, Harrison DW, Wright JW: A double-blind, placebo-controlled, randomized trial of the effects of dark chocolate and cocoa on variables associated with neuropsychological functioning and cardiovascular health: clinical findings from a sample of healthy, cognitively intact older adults. Am J Clin Nutr 2008, 87:872-880.

18. Grassi D, Desideri G, Necozione S, Lippi C, Casale R, Properzi G, Blumberg JB, Ferri C: Blood pressure is reduced and insulin sensitivity increased in glucose-intolerant, hypertensive subjects after 15 days of consuming high-polyphenol dark chocolate. J Nutr 2008, 138:1671-1676.

19. Grassi D, Lippi C, Necozione S, Desideri G, Ferri C: Short-term administration of dark chocolate is followed by a significant increase in insulin sensitivity and a decrease in blood pressure in healthy persons. Am J Clin Nutr 2005, 81:611-614.

20. Muniyappa R, Hall G, Kolodziej TL, Karne RJ, Crandon SK, Quon MJ: Cocoa consumption for 2 wk enhances insulin-mediated vasodilatation without improving blood pressure or insulin resistance in essential hypertension. Am J Clin Nutr 2008, 88:1685-1696.

21. Davison K, Coates AM, Buckley JD, Howe PR: Effect of cocoa flavanols and exercise on cardiometabolic risk factors in overweight and obese subjects. Int J Obes (Lond) 2008, 32:1289-1296.

22. Shiina Y, Funabashi N, Lee K, Murayama T, Nakamura K, Wakatsuki Y, Daimon M, Komuro I: Acute effect of oral flavonoid-rich dark chocolate intake on coronary circulation, as compared with non-flavonoid white chocolate, by transthoracic Doppler echocardiography in healthy adults. Int J Cardiol 2009, 131:424-429.

23. Ried K, Frank O, Stocks N: Dark chocolate or tomato extract for prehypertension: a randomised controlled trial. BMC Complement Altern Med 2009, 9:22.

24. Monagas M, Khan N, Andres-Lacueva C, Casas R, Urpi-Sarda M, Llorach R, Lamuela-Raventos RM, Estruch R: Effect of cocoa powder on the modulation of inflammatory biomarkers in patients at high risk of cardiovascular disease. Am J Clin Nutr 2009, 90:1144-1150.

25. Desch S: Effect of different dosages of dark chocolate on arterial blood pressure in cardiovascular high-risk patients. University of Leipzig, Leipzig, Germany 2009 [http://clinicaltrials.gov/ct2/show/NCT00661986].

26. Poulter NR: The effect of cocoa flavanoids on blood pressure. Imperial College, London, United Kingdom 2006 [http://clinicaltrials.gov/ct2/show/ NCT00125866].

27. Davison K, Berry NM, Coates AM, Buckley JD, Howe PRC: Is there a threshold effect of flavanol rich cocoa on blood pressure? Hypertension, Abstracts from the 30th Annual Scientific Meeting of the High Blood Pressure Research Council of Australia: Melbourne, Australia December 3-5, 20082009 53:1104-1105.

28. Cochrane Handbook for Systematic Reviews of Interventions. The Cochrane Collaboration. [http://www.cochrane.org/resources/handbook], accessed 24 March 08.

29. Review Manager (RevMan) [Computer program]. Version 5.0 Copenhagen: The Nordic Cochrane Centre, The Cochrane Collaboration, 2008. [http://www.cc-ims.net/RevMan], accessed 30 July 09.

30. Ried K, Frank OR, Stocks NP, Fakler P, Sullivan T: Effect of garlic on blood pressure: a systematic review and meta-analysis. BMC Cardiovasc Disord 2008, 8:13.

31. StataCorp. 2007. Stata Statistical Software: Release 10. College Station, TX: StataCorp.

32. Begg CB, Mazumdar M: Operating characteristics of a rank correlation test for publication bias. Biometrics 1994, 50:1088-1101.

33. Egger M, Davey Smith G, Schneider M, Minder C: Bias in meta-analysis detected by a simple, graphical test. Bmj 1997, 315:629-634.

34. Allen RR, Carson L, Kwik-Uribe C, Evans EM, Erdman JW Jr: Daily consumption of a dark chocolate containing flavanols and added sterol esters affects cardiovascular risk factors in a normotensive population with elevated cholesterol. J Nutr 2008, 138:725-731.

35. Erdman JW Jr, Carson L, Kwik-Uribe C, Evans EM, Allen RR: Effects of cocoa flavanols on risk factors for cardiovascular disease. Asia Pac J Clin Nutr 2008, 17(Suppl 1):284-287.

36. Balzer J, Rassaf T, Heiss C, Kleinbongard P, Lauer T, Merx M, Heussen N, Gross HB, Keen CL, Schroeter H, Kelm M: Sustained benefits in vascular function through flavanol-containing cocoa in medicated diabetic patients a double-masked, randomized, controlled trial. J Am Coll Cardiol 2008, 51:2141-2149.

37. Faridi Z, Njike VY, Dutta S, Ali A, Katz DL: Acute dark chocolate and cocoa ingestion and endothelial function: a randomized controlled crossover trial. Am J Clin Nutr 2008, 88:58-63.

38. Almoosawi S, Fyfe L, Ho C, Al-Dujaili E: The effect of polyphenol-rich dark chocolate on fasting capillary whole blood glucose, total cholesterol, blood pressure and glucocorticoids in healthy overweight and obese subjects. Br J Nutr 2010, 103:842-850.

39. Adamson GE, Lazarus SA, Mitchell AE, Prior RL, Cao G, Jacobs PH, Kremers BG, Hammerstone JF, Rucker RB, Ritter KA, Schmitz HH: HPLC method for the quantification of procyanidins in cocoa and chocolate samples and correlation to total antioxidant capacity. J Agric Food Chem 1999, 47:4184-4188.

40. Singleton $\mathrm{VL}$, Rossi JA: Colorimetric of total phenolics with phosphomolybdic-phosphotungstic acid reagents. Am J Enology Viticulture 1965, 16:144-158.

41. Gu L, House SE, Wu X, Ou B, Prior RL: Procyanidin and catechin contents and antioxidant capacity of cocoa and chocolate products. J Agric Food Chem 2006, 54:4057-4061.

42. Wollgast J, Anklam E: Review on polyphenols in Theobroma cacao: changes in composition during manufacture of chocolate and methodolgy for identification and quantification. Food Research International 2000, 33:423-447.

43. Weir MR: Impact of age, race, and obesity on hypertensive mechanisms and therapy. Am J Med 1991, 90:3S-14S.

44. Wofford MR, Smith G, Minor DS: The treatment of hypertension in obese patients. Curr Hypertens Rep 2008, 10:143-150.

45. Reinhart KM, Coleman Cl, Teevan C, Vachhani P, White CM: Effects of garlic on blood pressure in patients with and without systolic hypertension: $a$ meta-analysis. Ann Pharmacother 2008, 42:1766-1771.

46. Glynn RJ, L'Italien GJ, Sesso HD, Jackson EA, Buring JE: Development of predictive models for long-term cardiovascular risk associated with systolic and diastolic blood pressure. Hypertension 2002, 39:105-110.

47. Huxley RR, Neil HA: The relation between dietary flavonol intake and coronary heart disease mortality: a meta-analysis of prospective cohort studies. Eur J Clin Nutr 2003, 57:904-908.

48. Minor DS, Wofford MR, Jones DW: Racial and ethnic differences in hypertension. Curr Atheroscler Rep 2008, 10:121-127.

\section{Pre-publication history}

The pre-publication history for this paper can be accessed here: http://www.biomedcentral.com/1741-7015/8/39/prepub

doi:10.1186/1741-7015-8-39

Cite this article as: Ried et al.: Does chocolate reduce blood pressure? A meta-analysis. BMC Medicine 2010 8:39.

\section{Submit your next manuscript to BioMed Central and take full advantage of:}

- Convenient online submission

- Thorough peer review

- No space constraints or color figure charges

- Immediate publication on acceptance

- Inclusion in PubMed, CAS, Scopus and Google Scholar

- Research which is freely available for redistribution 